\title{
Synchronous Frequency Support of Photovoltaic Power Plants with Inertia Emulation
}

\author{
Cristian Verdugo, Andres Tarraso, Jose I. Candela, Joan Rocabert \\ Department of Electrical Engineering \\ Polytechnic University of Catalonia \\ Terrassa, Spain \\ Email: cristian.andres.verdugo@upc.edu
}

\author{
Pedro Rodriguez \\ Department of Electrical Engineering \\ Loyola Andalucia University \\ Seville, Spain \\ Email:prodriguez@uloyola.es
}

\begin{abstract}
Grid stability is one of the main concerns in renewable energies. The lack of inertia and their low capability to provide frequency support has created the need for implementing new control strategies to solve this problem. In current networks, frequency and voltage support are performed through synchronous generators, which provide an inherent grid support due to the inertia presented in their mechanical rotors. Based on the same concept, renewable energies based on power converters have introduced synchronous controllers to emulate the dynamic behavior of synchronous generators and provide voltage and frequency support. However, most synchronous control strategies integrate their controllers as an add-on firmware embedded in each power converter, without presenting a coordinated synchronous performance when several converters operate in a PV power plant. The aggregation of several power converters operating with a coordinated synchronous response would be advantageous in these cases, since they can provide a harmonic response with an automatic power distribution when grid support is required. This paper presents a synchronous control strategy for photovoltaic power plants, which manages several power converters as an aggregated synchronous system.
\end{abstract}

Index Terms-Grid frequency support, Photovoltaic Power Plants, Synchronous Power Control.

\section{INTRODUCTION}

Photovoltaic (PV) power plants used to operate at maximum power levels, but their high penetration and the variability in their primary sources due to uncertain weather conditions has demanded that PV power plants provide frequency and voltage support. Power variations may generate frequency oscillations in weak grids and consequently, instability in the network. However, it is well known that frequency stability is performed by Synchronous Generators (SG), which provide power support through the inertia in their mechanical rotors. In order to provide frequency support, PV power plants require external control strategies and energy storage systems, since they are not able to provide support by themselves.

Several strategies have been introduced to deal with this problem. Droop controllers adjust the power according to a frequency reference in order to increase or decrease the power supplied [1]. Other control strategies are based on Virtual Synchronous Machine (VSM) controllers, which emulate the electromechanical and the electrical interaction of a SG. In [2], the synchronverter controller is presented. This strategy emulates the electromechanical interaction of a SG and defines the current reference through the relationship between the stator flux and the mechanical torque. In [3], the impact of a virtual impedance is studied in terms of stability, dynamic performance and load adaptability in islanding mode. Another VSM strategy is the Synchronous Power Control (SPC), which synthesizes the electromechanical and the electrical interaction of a SG. The electromechanical characteristic regulates the inverter frequency through the virtual inertia and the damping coefficient, while the electrical characteristic emulates the stator through a virtual admittance to operate the converter as a controller current source [4]- [5], providing grid support under voltage oscillations, sags and harmonic compensation. This control strategy has proven to be an effective VSM for PV applications [6].

All control strategies mentioned are integrated independently in each power converter, without considering the possibility of controlling a cluster of converter as a single unit, providing grid frequency and voltage support in a certain point as a coordinated synchronous system. For this purpose, a Synchronous Central Controller for PV power plants based on the SPC architecture is presented. The control proposed manages several power converters as a single unit, emulating a sole SG at the point of common coupling $\left(P_{c c}\right)$. Therefore, the power required to provide frequency support is automatically shared between all power converters, giving rise to an aggregated virtual synchronous generator.

This paper is organized as follows: Section II introduces the current configuration of PV power plants. Then, in Section III the proposed control configuration and its architecture are described. In Section IV simulation results validates the control operation through a PV power plant connected to a weak grid. Finally Section V concludes the work.

\section{Photovoltaic Power Plant}

PV power plants have an architecture similar to the one illustrated in Fig.1. In this configuration, a group of PV converters are connected in parallel to a medium voltage (MV) collector and then connected to the transmission line through a step-up transformer. Depending on the rated power levels, several MV collectors are placed in the PV power plant. The power rating of these installations round between 0.1 to 1000 MW. 


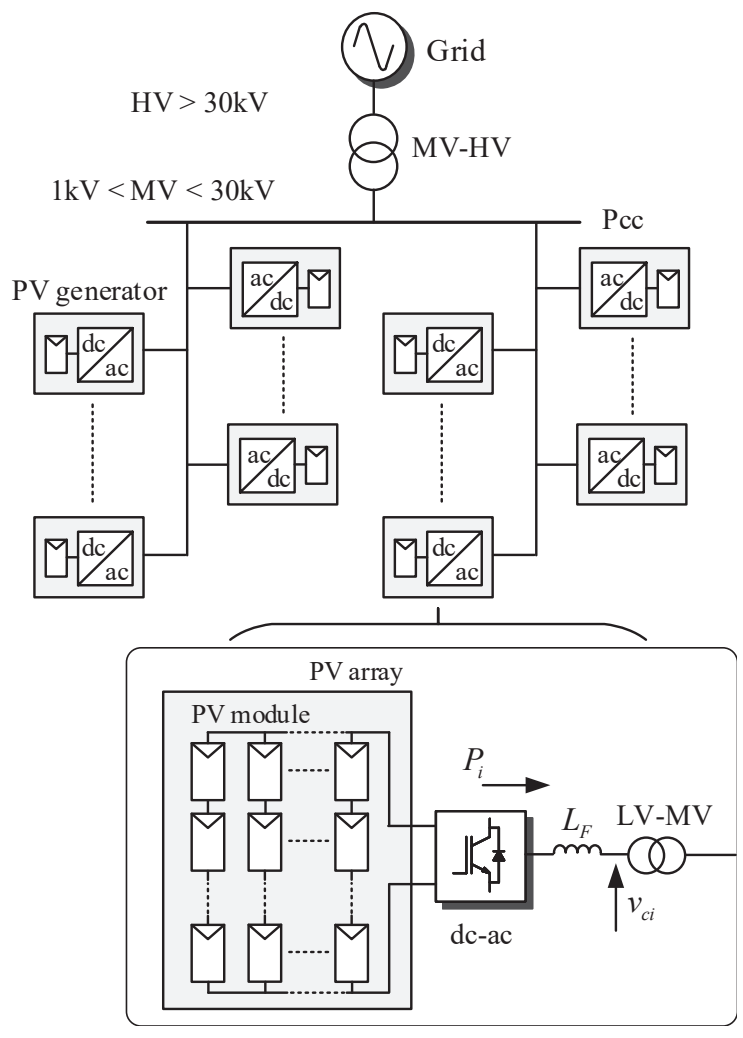

Fig. 1. PV power plant architecture

The PV generator represents a single power generation unit connected to the MV collector. A dc-ac power converter regulates the power generated by the $\mathrm{PV}$ modules to operate either at maximum power level or with power curtailment. The operation mode will be defined according with the grid requirements and the auxiliary services to provide voltage and frequency support. The PV modules are distributed in series and parallel connection to achieve the voltage and current levels required in the dc side. Normally, the dc voltage ranges between $700 \mathrm{~V}$ and $1200 \mathrm{~V}$. Furthermore, the ac converter side is connected to a filter to eliminate high frequency components and thus reduces the current ripple.

Regarding the control architecture, PV power plants are controlled through two control levels: the central controller at plant level and the local controller in the PV generator. The central controller (higher layer) manages the global active and reactive power reference and it defines the power level required by all PV generators through participation factors [7], which associate the power in the PV generators with the total power in the PV plant. Besides, this central controller regulates the voltage at $P_{c c}$ through a reactive power controller. The local controller operates at lower level and it controls the ac current of each PV generator according with the power references received from the central controller.

Currently, PV power plants are claimed to provide voltage and frequency support. Therefore, control strategies such as the SPC have been introduced in power converters to support the grid. This control strategy is embedded locally in each PV generator, emulating virtual inertia while providing a damped response. However, the grid support is given at the connection point of each PV generator and not necessary this support represent the level expected at $P_{c c}$. For this reason, the synchronous central controller introduces a new concept of VSM, which emulates the entire PV plant as a sole SG, where the electromechanical part is emulated at $P_{c c}$, while maintaining the synchronous characteristic in all PV generators.

\section{SYNCHRONOUS CENTRAL CONTROLLER}

The synchronous central controller emulates a particular $\mathrm{SG}$, since it introduces the concept of a single virtual rotor emulated at $P_{c c}$ with multiple stators emulated in each PV generator. As a result, the inertia and damped response are emulated at $P_{c c}$, while the power distribution is performed automatically by each PV generator. The synchronous central controller can be divided in two control layers: the higher control layer introduces the electromechanical model of a SG, acting somehow as a virtual rotor with a specific electromotive force (emf), $e$. Instead, the lower control layer emulates a virtual stator with a local emf, $e_{i}$, which is used to determine the appropriate power level provided by each PV generator at the connection point.

The advantage of this control architecture relies on the capability of providing an automatic power distribution among all PV converters connected, while providing a specific inertia and damped response at $P_{c c}$. The electrical representation of a PV power plant based on the synchronous central controller is illustrated in Fig.2.

As stated before, each PV generator emulates a virtual stator represented by an electromotive force with an appropriate amplitude, $E$, and phase angle, $\theta_{E}$, in series with an impedance $Z_{v}=R_{v}+j X_{v}$ [4]. All PV generators are connected to a common ac collector through an impedance $Z_{l}$, which represents the equivalent impedance between the ac filter, the LV-MV transformer and the transmission line.

According to the SG model presented in [8], the total impedance in a high power system can be considered inductive, hence, the electrical power provided from one PV generator to the grid can be written as.

$$
P_{i}=\frac{E_{i} V_{g}}{X_{i}} \sin \left(\theta_{E i}-\theta_{g}\right)=\frac{E_{i} V_{g}}{X_{i}} \sin \left(\delta_{i}\right)
$$

where $X_{i}$ is the equivalent reactance of one PV generator and $\delta_{i}$ is the angle difference between $\theta_{E i}$ and $\theta_{g}$. This angle is also known as the load angle and can be derived from the rotor emulated at $P_{c c}$.

\section{A. Inertia analysis}

The load angle is related to the frequency deviation of the rotor position in a SG. According to [8], the dynamic behavior of this angle can be studied through the swing equation presented in (3). 


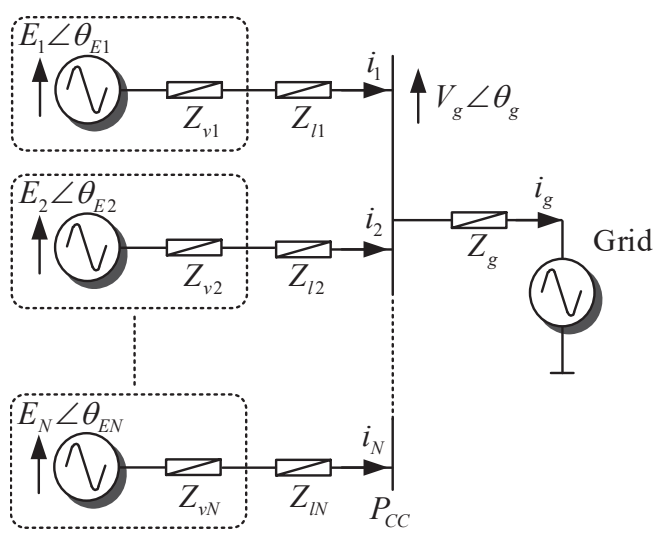

PV Generator

Fig. 2. Electrical representation of a PV power plant based on the synchronous central controller.

$$
\begin{aligned}
\frac{d \delta_{m}}{d t} & =\Delta \omega_{r} \\
J \frac{d \Delta \omega_{r}}{d t} & =\frac{P_{m}-P_{e}}{\omega_{B}}-D \Delta \omega_{r}
\end{aligned}
$$

where $P_{m}$ and $P_{e}$ are the mechanical and the electrical power, $\omega_{r}$ is the angular speed deviation of the rotor, $J$ is the moment of inertia, $\omega_{B}$ is the base frequency and $D$ is the damping constant. The moment of inertia can also be represented in terms of the inertia constant $H$ by means of.

$$
J=\frac{2 H S_{N}}{\omega_{B}^{2}}
$$

where $S_{N}$ is the nominal power of the SG.

The expressions (2) to (4) are used by the electromechanical interaction of the SPC strategy to determine the phase angle of the emf and thus control the active power, while a reactive power controller is implemented to control the emf amplitude. As a result, a single power converter unit emulates the inertia response of a SG.

\section{B. General control architecture}

The synchronous power controller contemplates the same concept of the SPC. However, in order to provided a coordinated synchronous action between all power converters, the control is divided in two control layers. The central control regulates the total power at $P_{c c}$ and emulates inertia to support the grid. Instead, the local control defines the local electromotive force needed to generate the appropriate current references in the inner control loop.

In Fig. 3 the control architecture of the synchronous central controller illustrates how the central and the local control interact. The central control generates the appropriate emf deviation, $\Delta E$, and the load angle required at $P_{c c}$ to provide the correct grid support. The local control is divided in three control blocks: the load angle regulation block is in charge

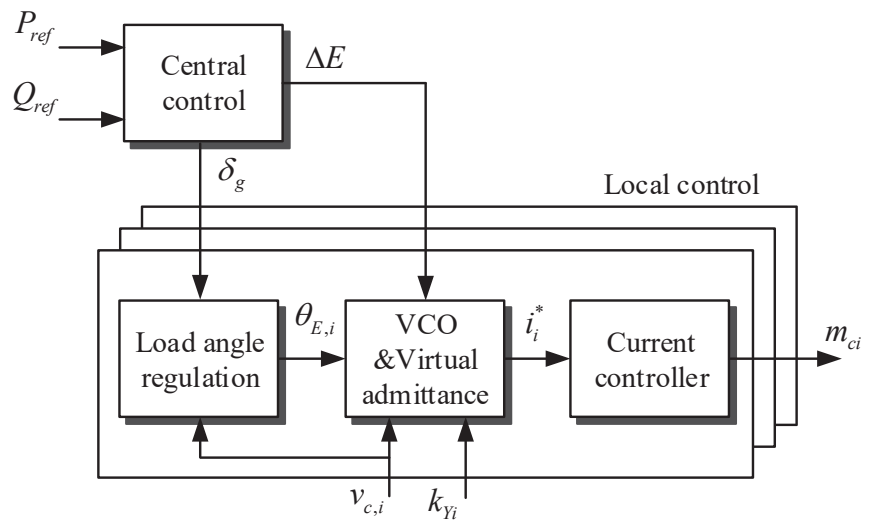

Fig. 3. Control architecture of the synchronous central controller.

of setting the phase angle $\theta_{E, i}$ for the local emf, the virtual admittance block defines the ac current reference through the voltage difference between the local emf and the voltage at the connection point of the PV generator, and the current controller generates the modulation index for controlling the power converter.

\section{Central control}

The central control regulates the power exchange with the electrical grid through the electromechnical interaction. Fig.4 illustrates the control structure of the central control, where the active power is commanded by the load angle, $\delta_{g}$. The transfer function $\mathrm{H}_{\mathrm{M}}$ links the active power with the synchronous frequency deviation, $\Delta \omega$, which is added to the base frequency and then integrated to provide the phase angle, $\theta_{E}$, of the emf at $P_{c c}$. The transfer function $\mathrm{H}_{\mathrm{M}}$ is designed based on the swing equation (3).

$$
\frac{\Delta \omega}{\Delta P}=\frac{1}{\omega_{B}(J s+D)}
$$

In a real $\mathrm{SG}$, the previous expression represents the interaction of the active power exchange and the kinetic energy of the rotor. In the case of the synchronous central controller, the active power balance acts on the dc voltage and the primary source of each PV generator unit.

The structure and the control parameters of $\mathrm{H}_{M}$ can be calculated based on the electromechanical analysis presented in [5].

To distribute the active power among all PV generators, we need to calculate the load angle $\delta_{g}$ at $P_{c c}$. In order to do that, the phase angle of the virtual rotor is used to rotate the voltage $v_{g}$ in a new virtual synchronous reference frame (VSRF). This rotation is performed through the Park's transformation by means of.

$$
\begin{aligned}
v_{g, d q E} & =\hat{v}_{g} \cos \left(\delta_{g}\right)-j \hat{v}_{g} \sin \left(\delta_{g}\right) \\
& =v_{g, d E}-j v_{g, q E}
\end{aligned}
$$




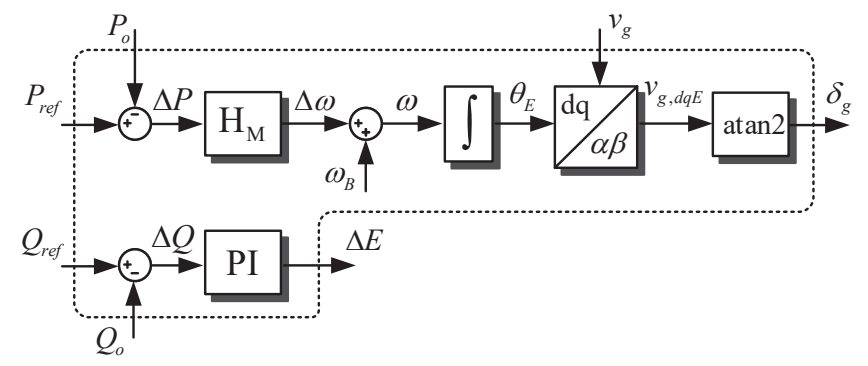

Fig. 4. Central control structure

The previous expression holds the $d$ component of $v_{g}$ in phase with the $d$ component of the emf. Once the grid voltage is projected in this VSRF, the arctangent between the $q$ and $d$ components determines the load angle.

$$
\delta_{g}=\tan ^{-1}\left(\frac{v_{g, q E}}{v_{g, d E}}\right)
$$

The reactive power control defines the emf deviation $\Delta E$ to provide voltage support at $P_{c c}$. The power regulation is performed through a PI controller.

\section{Local control}

The load angle and the emf amplitude are used in the local control to determine the active and reactive power generated by each PV converter. As mentioned, the local control is divided in the load angle regulation, the virtual admittance and the current controller. In Fig.5, the load angle regulation defines the phase angle, $\theta_{E, i}$ and its reference is the central load angle $\delta_{g}^{*}$. Note that a voltage limiter based on [9] is used to curtail the power at the maximum level available. The error between $\delta_{g}$ and $\delta_{E, i}$ is processed through a PI controller and then the frequency $\omega_{i}$ is integrated to generate $\theta_{E, i}$. The purpose of this control block is to maintain the same load angle in all PV generators, regardless their location in the PV power plant.

The voltage controller oscillator (VCO) generates the threephase sinusoidal waveform of the local emf, $e_{i}$. The emf amplitude is provided by the central control and the phase angle from the load angle regulation. Subsequently, the local emf is compared with the voltage $v_{c i}$ measured at the ac side and the difference is processed through the virtual admittance to set the current reference. The virtual admittance results from the virtual impedance $\left(1 / Z_{v}\right)$ in the stator and it is required to operate the power converter as a controlled current source [6].

$$
Y_{i}=\frac{k_{Y i}}{L_{v i} s+R_{v i}}
$$

Equation (8) introduces the virtual admittance structure, where $L_{v}$ and $R_{v}$ represent the virtual inductance and the virtual resistance respectively. The parameter $k_{Y i}$ is the participation factor and it is defined by the relationship between the power level at $P_{c c}$ and the power produced by the PV generator.
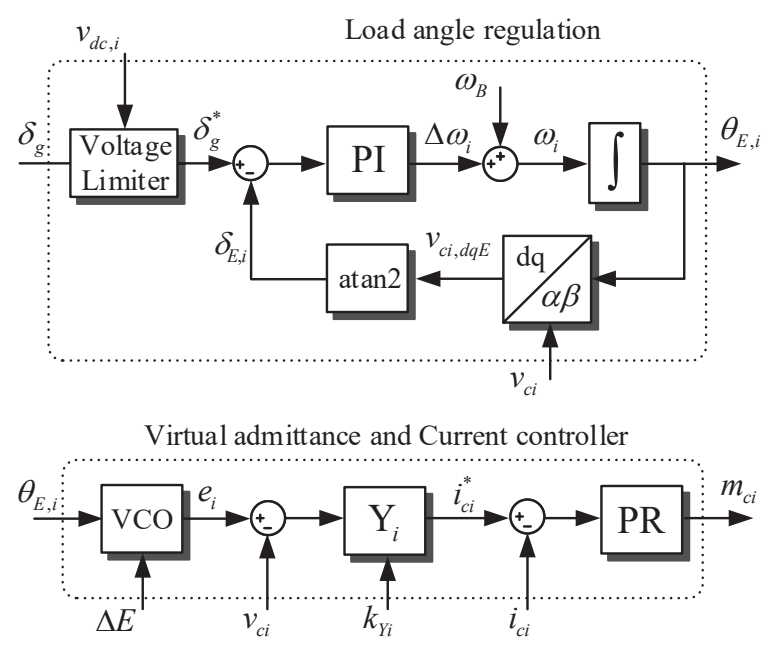

Fig. 5. Local control structure

$$
k_{Y i}=\frac{P_{i, p u}}{\sum_{i=1}^{N} P_{i, p u}}, \quad N=\sum_{i=1}^{N} k_{Y 1}
$$

Equation (9) defines the participation factor for all PV generators. Note that the addition of all participation factors must add the number of power converters connected.

The current control loop regulates the ac current of each PV generator according with the reference provided from the virtual admittance. The dynamic response of one PV generator can be represented through the following expression.

$$
L_{f i} \frac{d i_{i}}{d t}+R_{f i} i_{i}=v_{o i}-v_{c i}
$$

where $i_{i}$ is the current at the ac side, $v_{o i}$ is the voltage at the ac terminals in the power converter, $L_{f i}$ is the filter inductance and $R_{f i}$ the filter resistance.

\section{Simulation Results}

The objective of the simulation re0sults is to verify the power distribution and the grid frequency support of the synchronous central controller. Fig.6 shows the setup configuration, where the PV power plant is formed by three $2 \mathrm{~L}-\mathrm{VSC}$ connected at $P_{c c}$ through ac filters and step-up transformers. The maximum power and the open circuit operation of the PV array is represented by a dc source in series connection to a dc resistor. The grid is simulated using a synchronous generator such as described in [8], with low inertia and a frequency controller to return the frequency to its nominal value after a power variation happens. Simulation and control parameters are listed in Table.I.

In order to provide grid frequency support, the PV power plant represents $20 \%$ of the rated power at $P_{c c}$ and it operates at $88 \%$ of its nominal power, e.g emulating a curtailment situation. A resistive load of $4 \%$ of the rated power is connected 


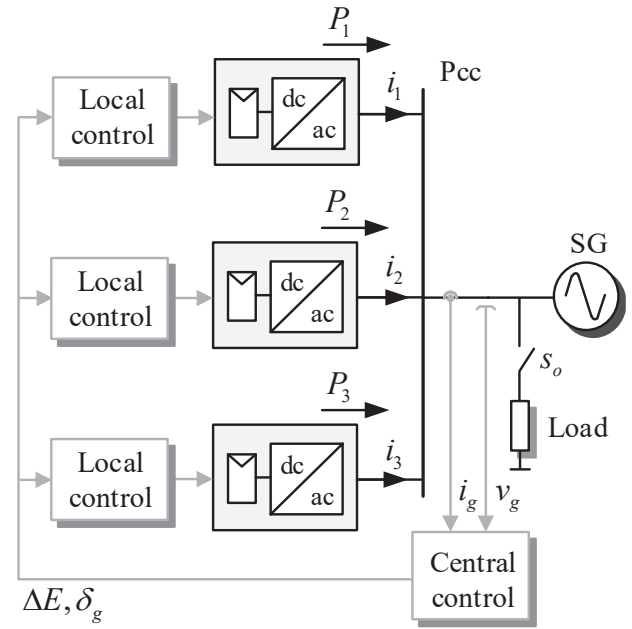

Fig. 6. Setup configuration of simulation results

to increase the power demanded and generates a frequency perturbation. The participation factors are defined in such a way that the PV generator 1 provides $36 \%$ of the total power generated, the PV generator $233 \%$ and the PV generator 3 $31 \%$.

In the study case, the PV plant provides grid frequency support under different inertia values. Fig.7 illustrates the grid frequency response when the PV plant has an inertia of $1 \mathrm{~s}$, $5 \mathrm{~s}$ and $10 \mathrm{~s}$ respectively. When the load is connected, we can appreciate that as the inertia increases, the frequency support

TABLE I

Simulation PARAMETERS

\begin{tabular}{lcc}
\hline \hline Parameters & Symbol & Value \\
\hline \hline PV power plant parameters & & \\
\hline Rated power at $P_{c c}$ & $P_{N}$ & $30 \mathrm{MW}$ \\
Nominal power per PV generator & $P_{M}$ & $2 \mathrm{MW}$ \\
Number of PV generators & $N$ & 3 \\
Grid voltage rms (line-to-line) & $v_{g}$ & $33 \mathrm{kV}$ \\
Grid frequency & $f_{S}$ & $50 \mathrm{~Hz}$ \\
dc source & $V_{d c}$ & $800 \mathrm{~V}$ \\
dc voltage at maximum power & $V_{m}$ & $636 \mathrm{~V}$ \\
dc-link capacitance & $C_{d c}$ & $2.2 \mathrm{mF}$ \\
dc resistor & $R_{d c}$ & $52 \mathrm{~m} \Omega$ \\
Switching frequency & $f_{c}$ & $2.2 \mathrm{kHz}$ \\
\hline Transformer voltage & $V_{T}$ & $400 \mathrm{~V}-33 \mathrm{kV}$ \\
Transformer power & $P_{T}$ & $2 \mathrm{MW}$ \\
\hline Per unit base & & \\
\hline Base voltage & $V_{B}$ & $27 \mathrm{kV}$ \\
Base power & $S_{N}$ & $6 \mathrm{MW}$ \\
Base current & $I_{B}$ & $148 \mathrm{~A}$ \\
\hline Control parameters & & \\
\hline Participation factor converter 1 & $k_{Y 1}$ & 1.058 \\
Participation factor converter 2 & $k_{Y 2}$ & 1 \\
Participation factor converter 3 & $k_{Y 3}$ & 0.941 \\
Virtual inductance & $L_{v i}$ & $0.1 \mathrm{pu}$ \\
Virtual resistance & $R_{v i}$ & $0.03 \mathrm{pu}$ \\
\hline \hline & & \\
\hline & & \\
\hline
\end{tabular}
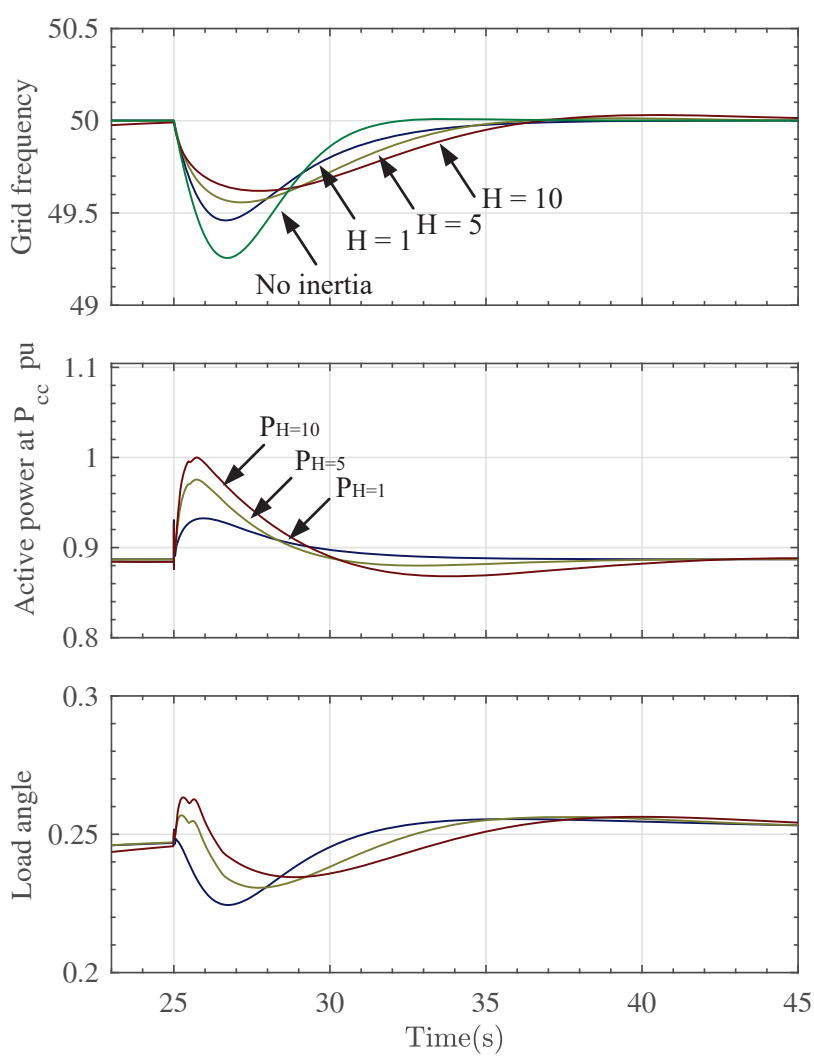

Fig. 7. Grid frequency, output power and load angle at $P_{c c}$ under inertia emulation $H=1 s, 5 s, 10 s$.

increases as well. This behavior can also be observed in the total active power, due to the fact that the inertia is directly related to the active power. With inertia $H=10$, the PV plant provides its nominal power and the frequency decrease until 49.64 Hz. Remember that the central load angle is responsible for changing the set point of the PV generators in order to provide the appropriate active power. The load angle is shown in Fig.7.

All PV generators operate at different power levels. In consequence, when the power support increases, the PV generator with the higher participation factor generates the higher power level. However, the maximum affordable power is limited by the minimum acceptable dc voltage level. In the local control, a dc voltage limiter has been integrated to avoid power request beyond the maximum power. Therefore, when PV generators reach their minimum dc voltage level, the power is curtailed. Fig.8 illustrates the power response of each PV generator when the PV plant has an inertia $H=10 s$. Since, the PV generator 1 reaches its minimum dc voltage level (at $0.795 \mathrm{pu}$ ) its power is limited and the rest of PV generators increase their power to support the grid when the load is connected. Despite the PV generator 1 limits its power, the PV plant automatically distribute the power sharing between the rest of converters.

To prove the current operation and grid synchronization, the three-phase voltage, the grid current and the converter currents 

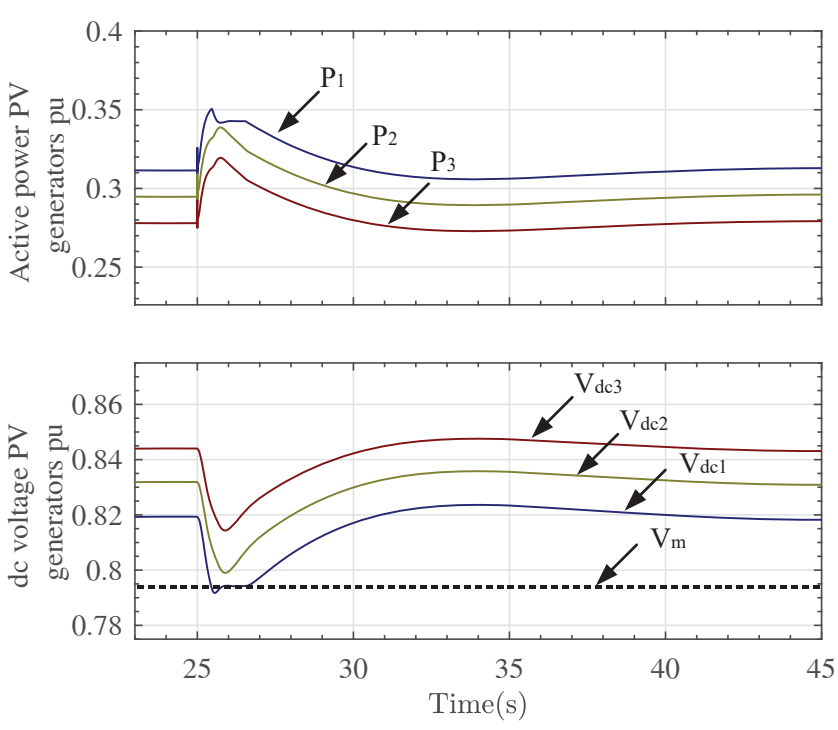

Fig. 8. Output power and dc voltage of each PV generator with inertia emulation $H=10 \mathrm{~s}$.
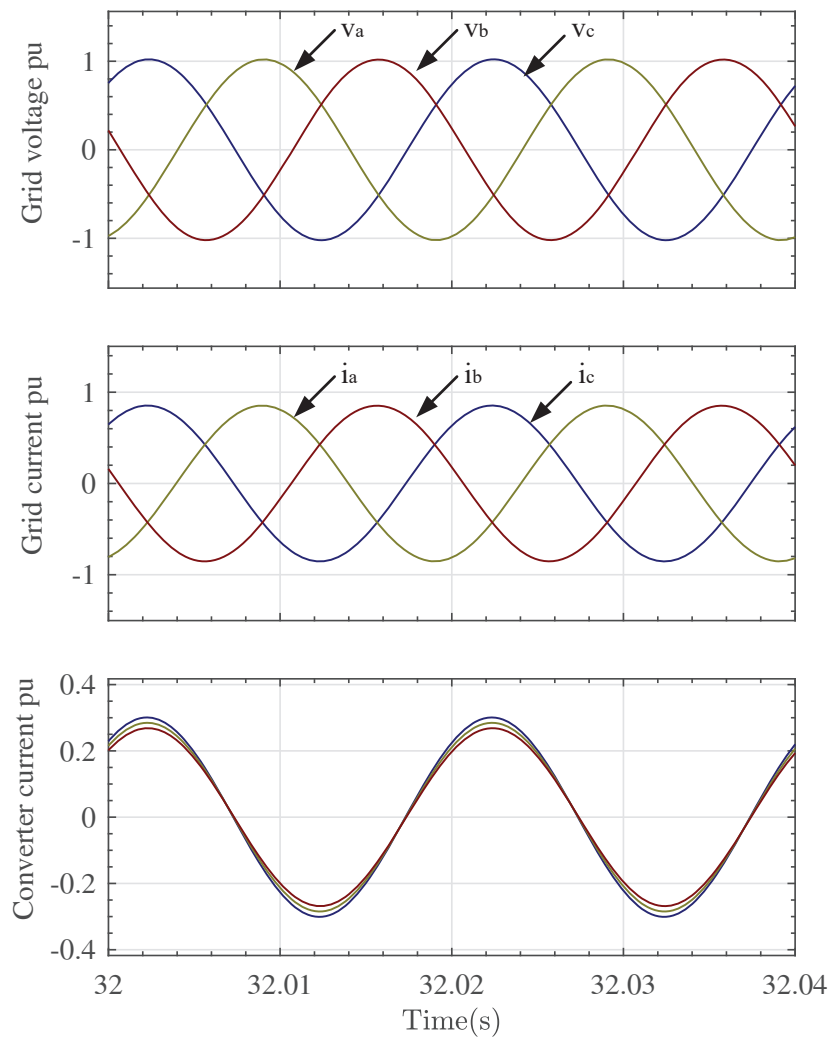

Fig. 9. Grid voltage, grid current and converter current with inertia emulation $H=10 s$.

provided by each PV generator are shown in Fig.9. We can appreciate how the grid voltage and current are synchronized, while the converter currents are proportional to the power generated.

\section{CONCLUSION}

Power converters based on synchronous control strategies are able to provide grid support functionalities in renewable energies. So far, synchronous controller have been embedded locally in each power converter unit, providing inertia and damping response. However, the grid support is given at the connection point of each PV generator and not at plant level. For this reason, the synchronous central controller for PV power plants has been presented. This controller is able to manage several PV generators as an aggregated system, emulating inertia through several interconnected and coordinated converters. The main idea is to emulate the rotor behavior of a SG at $P_{c c}$, while the virtual stators are emulated in the local control of each PV generator. Simulation results illustrate how higher inertia values provide higher frequency support under a perturbation at $P_{c c}$. Moreover, the automatic power distribution shows how different participation factors distribute the power demanded even though one PV generator achieves its maximum power.

\section{ACKNOWLEDGMENT}

This work was supported by the European Commission under project FLEXITRANSTORE - H2020-LCE-2016-2017SGS-774407 and by the Spanish Ministry of Science Innovation and Universities under the project FLEXIGRID RTI2018100921-B-C21. Any opinions, findings and conclusions or recommendations written in this work are those of the authors and do not necessarily reflect those of the host institutions and funders.

\section{REFERENCES}

[1] J. Liu, Y. Miura, and T. Ise, "Comparison of dynamic characteristics between virtual synchronous generator and droop control in inverter-based distributed generators," IEEE Transactions on Power Electronics, vol. 31, no. 5, pp. 3600-3611, May 2016.

[2] Q. Zhong and G. Weiss, "Synchronverters: Inverters that mimic synchronous generators," IEEE Transactions on Industrial Electronics, vol. 58, no. 4, pp. 1259-1267, April 2011.

[3] Y. Tao, Q. Liu, Y. Deng, X. Liu, and X. He, "Analysis and mitigation of inverter output impedance impacts for distributed energy resource interface,' IEEE Transactions on Power Electronics, vol. 30, no. 7, pp. 3563-3576, July 2015.

[4] W. Zhang, A. M. Cantarellas, J. Rocabert, A. Luna, and P. Rodriguez, "Synchronous power controller with flexible droop characteristics for renewable power generation systems," IEEE Transactions on Sustainable Energy, vol. 7, no. 4, pp. 1572-1582, Oct 2016.

[5] C. Verdugo, J. I. Candela, and P. Rodriguez, "Grid support functionalities based on modular multilevel converters with synchronous power control," in 2016 IEEE International Conference on Renewable Energy Research and Applications (ICRERA), Nov 2016, pp. 572-577.

[6] P. Rodriguez, C. Citro, I. Candela, J. Rocabet, and A. Luna, "Flexible grid connection and islanding of spc-based pv power converters," in 2015 IEEE Energy Conversion Congress and Exposition (ECCE), Sep. 2015, pp. $450-459$.

[7] Z. Moradi-Shahrbabak, A. Tabesh, and G. R. Yousefi, "Economical design of utility-scale photovoltaic power plants with optimum availability," IEEE Transactions on Industrial Electronics, vol. 61, no. 7, pp. 33993406, July 2014.

[8] D. J. B. J. Machowski, J. Bialek, Power System Dynamics: Stability and Control. 2nd Edition, Wiley, 2008.

[9] A. Tarraso, J. I. Candela, J. Rocabert, and P. Rodriguez, "Synchronous power control for pv solar inverters with power reserve capability," in IECON 2017 - 43rd Annual Conference of the IEEE Industrial Electronics Society, Oct 2017, pp. 2712-2717. 